\title{
so \\ 3D-Space Wireless Power Transfer for Device Charging and Energy Management
}

\author{
${ }^{1}$ Nikita Gaikwad, ${ }^{2}$ Swapnil Ramgirwar, ${ }^{3}$ Shubham Waykos, ${ }^{4}$ P. P. Mane \\ ${ }^{1,2,3,4}$ Dept. of Electronics and Telecommunication, Modern Education Society's College of Engineering, Pune - India
}

\begin{abstract}
This paper discusses the architecture of wireless low power transmission system to charge the mobile phone wirelessly as well as to give effective solution for energy savings and utilization. It is simple, affordable and robust system that can be easily installed and operated in order to solve certain real time power issues. Wireless power transmission is an emerging technology that is gaining increased visibility in recent years. Wireless power transmission holds a promising future for generating a small amount of electrical power to charge mobile wirelessly. Growing importance in wireless field which has numerous benefits such as it would completely eliminate the need of carrying charger along with mobiles. It would also help to reduce complexity caused due to wires. The phenomenon for energy generation incorporated in here is to use a renewable source. Solar panels are incorporated in this system as they play a key factor in providing a renewable source of energy on contrary to non-renewable or AC source supplies. The panels convert light into electrical energy and stored in batteries which is then used to charge mobile phone wirelessly. In this paper we are also trying to implement real time power monitoring and controlling system for home appliances such as fans, bulbs, etc. in robust way. Controlling of electrical devices can be performed by programming the main controller.
\end{abstract}

Keywords-WPT (Wireless Pawer Transfer), PV Array, LPC2148, Ocillator, CMRS (Coupled Magnetic Resonance System)

\section{INTRODUCTION}

Nikola Tesla had invented first radio and proved that he is the father of wireless communication. Nikola Tesla had first designed the idea of Wireless Power Transmission and demonstrated the transmission of electrical energy without wires. Wireless power transmission (WPT) is an efficient way for the transmission of electric power from one point to another through vacuum or atmosphere without the use of wire or any physical material. By using WPT, power can be transmitted using inductive coupling for short range, resonant induction for mid-range and Electromagnetic Wave power transfer. By using this technology, it is possible to supply power to places, which is hard to do using conventional wires. Currently, the use of inductive coupling is in development and research phases. The most common wireless power transfer technologies are the electromagnetic induction and the microwave power transfer. For efficient midrange power transfer, the wireless power transfer system must satisfy three conditions: (a) high efficiency, (b) large air gap, (c) high power. The microwave power transfer has a low efficiency. For near field power transfer this method may be inefficient, since it involves radiation of electromagnetic waves. Wireless power transfer can be done via electric field coupling, but electric field coupling provides an inductively loaded electrical dipole that is an open capacitor or dielectric disk. External objects may provide a relatively strong influence on electric field coupling. Magnetic field coupling may be preferred, since external objects in a magnetic field have the same magnetic properties as empty space. Electromagnetic induction method has short range. Since magnetic field coupling is a nonradiative power transfer method, it has higher efficiency. However, power transfer range can be increased by applying magnetic coupling with resonance phenomenon applied on. A magnetic field is generated when electric charge moves through space or within an electrical conductor.

Solar energy is energy obtained from the Sun. It is renewable, inexhaustible and environmental pollution free. Solar charged battery systems provide power supply for complete 24 hours a day irrespective of bad weather. By adopting the appropriate technology for the concerned geographical location, we can extract a large amount of power from solar radiations. More over solar energy is expected to be the most promising alternate source of energy. Hence solar panels are used here to generate power and stored it in batteries. The stored energy is then used to charge mobile phone wirelessly as well as to supply energy for other home appliances. In order to avoid unnecessary use of energy, effective energy saving technique is used by using ARM7 LPC2148 microcontroller. To improve the easiness and efficiency of electrical appliances have been the main motivation throughout this paper.

In section [II], explained the work done on the solar energy generation, power management and WPT till date. In section [III], design and working of overall system is elaborated. In section [IV], mathematical 
formulations are represented. Section number [V] shows advantages of the system as well as enlighten the future scope of this work.

\section{LITERATURE REVIEW}

Lots of work has been done on solar energy generation as well as on effective power management techniques till now. The paper [1] presents a solution for new green resource allocation problem using hybrid powering of communication systems from renewable sources. The power saving module switches the appliances in the room based on the presence of the person. It also controls the power delivered to fan and light according to temperature of room and natural daylight intensity [3].

Researchers at MIT in 2007 proposed the technique of Strongly Coupled Magnetic Resonance (SCMR) and attracted the world's attention by importance of its midrange, nonradiative and high-efficiency power transfer. In this paper [7], current developments and research progress in the SCMR area are presented. Advantages of SCMR are analysed by comparing it with the other wireless power transfer (WPT) technologies.

The topic of Inductive power transfer has been studied by many researchers all around the world and presently it is one of the hot topic among the researchers. It has been known that as the distance increases between primary side and secondary side, transfer efficiency decreases and thus a better contactless transfer control mechanism and selection of good quality metal with high mutual coefficient is required. Ran Tao, Salem, Hamdi, "Adaptive Relaying Protocol for Wireless Power Transfer and Information Processing" proceeding of the IEEE vol. 20, no. 10, October 2016, have given a detailed historical background, technological issues, and engineering applications of inductive power transfer in their paper. Sayyad, Sarvade, "Wireless Power Transmission for Charging Mobiles", vol. 12, June 2014 have designed a contactless battery charger for cellular phone using a monopole antenna. In his paper designed optimization has presented and they had also verified their result with experimental results.

\section{METHODOLOGY}

Figure 1 represents basic block diagram of overall system and its working. By means of solar panels electric energy is generated. This energy is provided to the entire system in order to run power management block as well as to charge the devices wirelessly. Use of solar energy in this work is due to its abundant availability which gives optimum solution for conventional methods of power generation which are not environmental free. Here use of wireless power transmission using inductive coupling is mean to reduce the wired complexity as well as improving way of living and it is not hazardous to human health due to use of frequency in $\mathrm{kHz}$ unlike microwave transmission which is in MHz. Power management block is added to represent a smart way for power saving and avoid unnecessary power wastage using ARM7 LPC2148 microcontroller. LPC2148 microcontroller requires very low power consumption to operate.

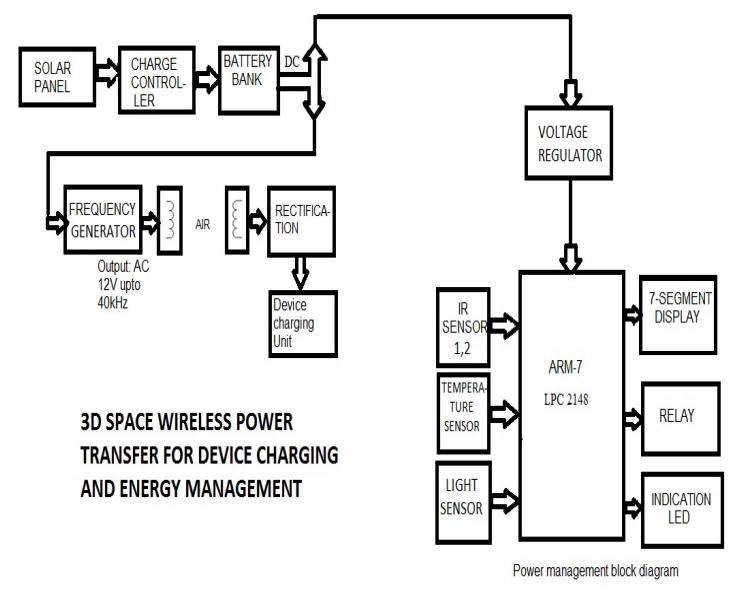

Fig.1 Block diagram of 3D space wireless power transfer for energy management and device charging

\section{Solar energy generation}

The solar panel is used to convert solar energy into electrical energy means in the form DC voltage. After the solar panel charge controller is used to control the constant voltage and constant current. In the charge controller circuit we are using the over voltage protection and Deep Discharge Protection. In the overcharge protection, opto-coupler is used to provide high electrical isolation. When the battery is fully charged then it automatically stop to charge and simultaneously the LED is blink and give indication that the battery is fully charged.

The deep discharge protection is very useful for avoiding battery to deep discharge. When the battery start to discharge, at threshold level it gives indication by blinking LED that the battery is discharged.

\section{Wireless power transfer}

Inductive or magnetic coupling works on the principle of electromagnetism. When a wire is near to a magnetic field, it induces a magnetic field in that wire. Transferring energy between wires through magnetic fields is inductive coupling. Magnetic resonant coupling uses the same principles as inductive coupling, but it uses resonance to increase the range at which the energy transfer can efficiently take place. Resonance can be two types: (a) series resonance \& (b) parallel resonance. In these both types of resonance, the principle of obtaining maximum energy is same but the methods are quite different. 
Wireless transmission technology has used in communication system from long time ago like mobile phones, dish TV etc. Frequency used in radio wave is very high i.e. in the range of $\mathrm{MHz}$. But frequency used in WPT used in electrical power application in the range of $\mathrm{KHz}$ so that it is not hazardous for human. This thesis presents use of WPT technology in mobile charger application. WPT mobile charger has several advantages over formal mobile charger. One of the most frequent failures in mobile charger is from the mechanical contact. To overcome from this type of problem WPT mobile charger can be used. Working principle behind WPT is electromagnetic induction phenomena. Since, in WPT technology air core is used so conversion efficiency is very low. To increase the efficiency of WPT, both primary and secondary coil should work on the resonant frequency. We are implementing primary series and secondary parallel resonant circuit for WPT.

\section{Power management}

For the intelligent power management we use LPC2148 microcontroller. The LPC2148 microcontroller are based on a 32 bit ARM7TDMI-S CPU with Real-time emulation and embedded trace support that combines the microcontroller with high speed flash memory ranging from $32 \mathrm{kB}$ to $512 \mathrm{kB}$. A 128 -bit wide memory interface and unique accelerator architecture enable 32-bit code execution at the maximum clock rate. For critical code size applications, the alternative 16-bit Thumb mode reduces code by more than $30 \%$ with minimal performance penalty. We also use IR sensor. This infrared sensor module is used for detecting reflecting surface. This sensor can be used to detect reflecting silver/white strip, obstacle detection, flame detection, etc. IR LED emits infrared radiation. This radiation illuminates the surface in front of LED. Surface reflects the infrared light. Depending on reflectivity of the surface the mount of light reflected varies. This reflected light is made incident on reverse biased IR sensor. When photons are incident on reverse biased junction of this diode, electron-hole pairs are generated, which results in reverse leakage current. Amount of electron-hole pairs generated depends on intensity of incident IR radiation. More intense radiation results in more reverse leakage current. This current can be passed through a resistor so as to get proportional voltage. Thus as intensity of incident rays varies, voltage across resistor will vary accordingly. This voltage can then be given to OPAMP based comparator. Output of the comparator can be read by microcontroller. Then relay is used for load purpose.

\section{MATHEMATICAL DESCRIPTION}

The mutual inductance $\mathrm{M}$ can be expressed as following

$\mathrm{M}=\mathrm{K} \times(\mathrm{LP} \times \mathrm{LS})^{1 / 2}$

Where $\mathrm{K}$ is the coupling coefficient which can be expressed as following
$\mathrm{K}=(1-(\mathrm{Lsc} / \mathrm{Lop}))^{1 / 2}$

Where Lsc means the value of measurement inductance from primary side when the secondary side is short circuited, and Lop means the value of measurement inductance from the primary side when the secondary side is open- circuit.

In order to simply the process of analysis, the line resistance of inductor and equivalent series resistance of capacitor will not be considered and resonant capacitor values of the primary side and the secondary side are required to analysis. The inductance value of the primary side and the secondary side can be obtained by the production of the induction coil with core, and decided the resonant frequency. The suitable value of capacitance can be calculated by

$\mathrm{Cp}=1 \div\left(\mathrm{w}^{2} \times\left(\mathrm{LP}-\left(\mathrm{M}^{2} / \mathrm{LS}\right)\right)\right)$

Where, $\mathrm{Cp}$ is primary coil capacitance, LP is primary coil inductance, $\mathrm{M}$ is mutual inductance, $\mathrm{w}$ is resonant frequency.

\section{RESULTS}

Proteus professional design combine the schematic capture and PCB layout program to provide a powerful, integrated and easy to use tools suite for education and professional PCB design. As a profession PCB design software with integrated shape based auto router, it provides features, such as fully featured schematic capture, highly configurable design, rules interactive SPICE circuit simulator, extensive, support for power planes, industry standard. Following images show the respective stimulated results obtained from Proteus software. We are trying to improve the power transfer distance to charge mobile phone without decreasing the efficiency.

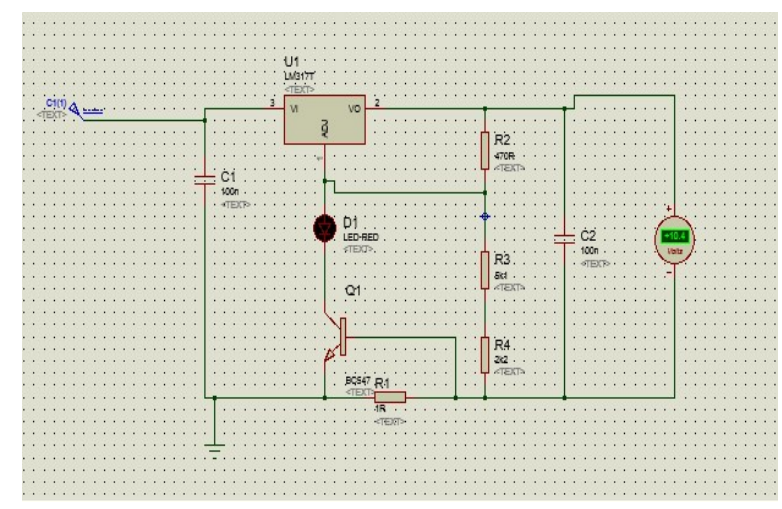

Fig.2 Stimulated circuit design of Charge controller 


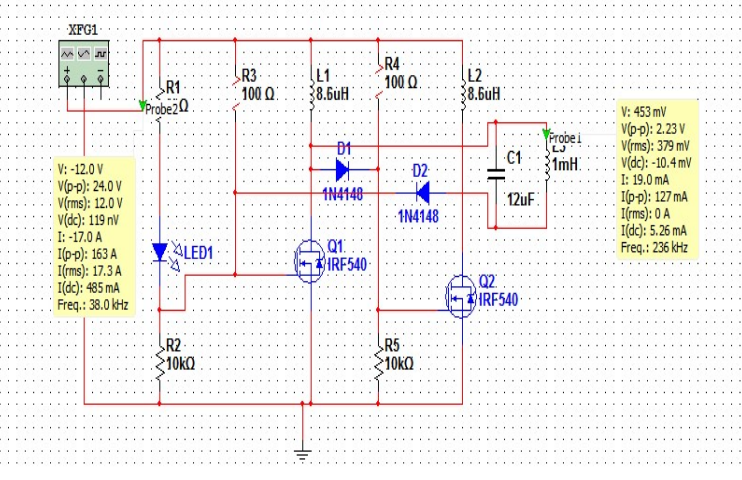

Fig.3 Stimulated cicrcuit design of Wireless Power Transmitter

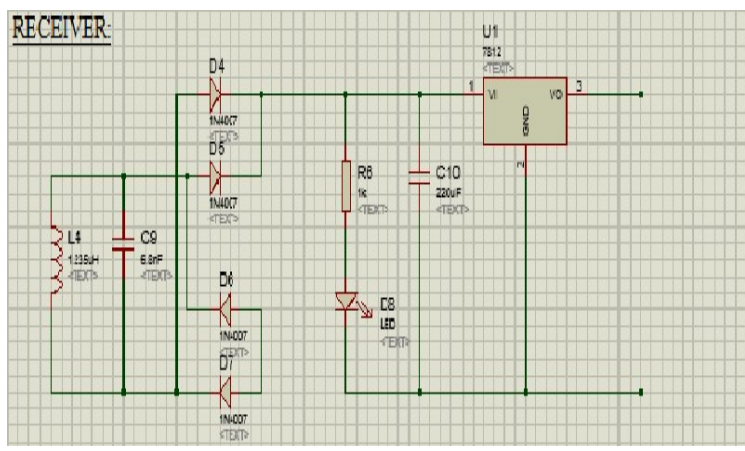

Fig. 4 Stimulated circuit design of Wireless Power Receiver

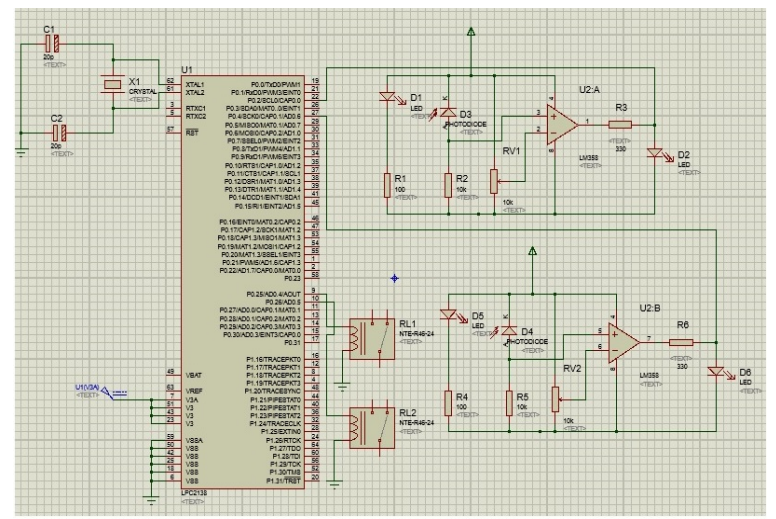

Fig.5 Stimulated cicrcuit design of Power Management using LPC2148 microcontroller

\section{FUTURE SCOPE}

The future work deals with finding ways to charge mobile battery without any charger chord with higher efficiency. The distance between mobile and battery can be extended up to $1 \mathrm{~m}$. Closed loop buck converter can be realized to get desired regulated DC output voltage. Dynamic wireless battery charger may also be designed. To get maximum energy transfer efficiency a suitable structure of inductor coil will be made.
This paper is very useful for minimizing the complicated wired networks for power transmission. There will be no need of wires to charge the devices like mobile, laptop, electric car, etc. and the devices can also be charged while moving. The threats generated due to wired power transmission such as shorting of wires, electric shocks, energy theft issues, etc. may be successfully avoided using this project. This system can be easily set up within any locations like home, colleges, hospitals, industries, etc. and the power generated from the solar energy sources can also be connected to main grid. Due to the use of solar energy source, pollution caused during power generation can be avoided to a great extent through this paper. This paper provides a smart way of power saving and avoids unnecessary wastage of electricity as well as related extra electricity billing charges by means of automatic power control system.

\section{CONCLUSION}

In this paper we tried to create energy from renewable energy sources like solar source due to which pollution can be avoided up to high extent. Electricity generated from these sources can be connected to main grid if required. This energy generated will be then used as power source for all the electric appliances within the locality. To save the energy by avoiding unnecessary power wastage, automatic power management and control system plays an important role in this paper. Thus there is no need to take care about power saving manually. With the advancements taking place in electrical and electronics world, it is now required to design efficient user friendly products using recent technologies. The paper not only focuses on effective energy generation and intelligent power management but also aims in wireless power transfer to charge the electric devices in efficient way. Although a lot of research is still in process for wireless power transmission, we tried to provide a wireless power transfer system for charging electric devices like mobile phones effectively. Now the user can charge their devices without using wires. The paper gives various smart solutions for certain power issues effectively and this system can be implemented anywhere like in homes, hospitals, colleges, libraries, industries, etc.

\section{REFERENCES}

[1] Taha Touzri, Mahdi Ben Ghorbel, Bechir Hamdaoui, Mohsen Guizani, "Efficient Usage of Renewable Energy in Communication Systems Using Dynamic Spectrum", IEEE transactions on wireless communications, vol. 15, no. 5, May 2016.

[2] Diego Arcos-Aviles, Julio Pascual, Luis Marroyo, "Fuzzy Logic-Based Energy Management System Design for Residential Grid-Connected Microgrids”, DOI 
10.1109/TSG.2016.2555245, IEEE Transactions on Smart Grid.

[3] R. Teixeira Pinto, M. Arag"u'es-Pẽnalba, "Optimal Operation of DC Networks to Support Power System Outage Management”, DOI 10.1109/TSG.2016.2586024, IEEE Transactions on Smart Grid.

[4] Ran Tao, Abdelhamid Salem, Khairi Ashour Hamdi, "Adaptive Relaying Protocol for Wireless Power Transfer and Information Processing", IEEE communications letters, vol. 20, no. 10, October 2016.

[5] Jinwook Kim, Do-Hyeon Kim, "Free-Positioning Wireless Power Transfer to Multiple Devices Using a Planar Transmitting Coil and Switchable Impedance Matching Networks", IEEE transactions on microwave theory and techniques, 2016.

[6] Zhu Liu, Zhizhang (David) Chen, "A Planar LShape Transmitter for Wireless Power Transfer
System”, DOI 10.1109/LAWP.2016.2615112, IEEE Antennas and Wireless Propagation Letters, 2016.

[7] Olutola Jonah, Stavros V. Georgakopoulos, "Orientation Insensitive Power Transfer by Magnetic Resonance for Mobile Devices”, 2013.

[8] M. Virili, F. Alimenti, L. Roselli, "Organic Frequency Doubler RFID Tag Exploiting 7.5MHz Wireless Power Transfer”, 2013.

[9] Aakib J. Sayyad, N. P. Sarvade, "Wireless Power Transmission for Charging Mobiles”, Jun 2014

[10] Tarique Salat, Shilpak Raich, "A Wireless Battery Charger for Mobile Device”, June 2013

[11] Sherif Hekal, Adel B. Abdel-Rahman, "Compact Wireless Power Transfer System Using Defected Ground Bandstop Filters", OCTOBER 2016 